\title{
Changes in glycemic control from 1996 to 2006 among adults with type 2 diabetes: a longitudinal cohort study
}

\author{
Karen J Blumenthal', Mary E Larkin', Gail Winning', David M Nathan 1,3 and Richard W Grant*1,2,3
}

\begin{abstract}
Background: Our objectives were to examine temporal changes in $\mathrm{HbA} 1 \mathrm{c}$ and lipid levels over a 10-year period and to identify predictors of metabolic control in a longitudinal patient cohort.

Methods: We identified all adults within our hospital network with T2DM who had HbA1c's measured in both 1996 and 2006 (longitudinal cohort). For patients with no data in 2006, we used hospital and social security records to distinguish patients lost to follow-up from those who died after 1996. We compared characteristics of the 3 baseline cohorts (longitudinal, lost to f/u, died) and examined metabolic trends in the longitudinal cohort.

Results: Of the 4944 patients with HbA1c measured in 1996, 1772 (36\%) had an HbA1c measured in 2006, 1296 (26\%) were lost to follow-up, and 1876 (38\%) had died by 2006. In the longitudinal cohort, mean HbA1c decreased by $0.4 \pm$ $1.8 \%$ over the ten-year span (from $8.2 \% \pm 1.7 \%$ to $7.8 \% \pm 1.4 \%$ ) and mean total cholesterol decreased by $49.3( \pm 46.5$ ) $\mathrm{mg} / \mathrm{dL}$. In a multivariate model, independent predictors of $\mathrm{HbA} 1 \mathrm{c}$ decline included older age (OR 1.41 per decade, 95\% Cl: 1.3-1.6, p < 0.001), baseline HbA1c (OR 2.9 per 1\% increment, $2.6-3.2, p<0.001)$, and speaking English (OR 2.1, 1.4$3.1, \mathrm{p}<0.001)$.

Conclusions: Despite having had diabetes for an additional 10 years, patients in our longitudinal cohort had better glycemic and cholesterol control in 2006 than 1996. Greatest improvements occurred in patients with the highest levels in the baseline year.
\end{abstract}

\section{Background}

As the incidence of diabetes continues to increase in the United States, it has become increasingly important to understand trends in progression of the disease over time [1]. Type 2 diabetes (T2D) is seen as an inexorably progressive disease, with beta cell failure and increasing peripheral insulin resistance leading to worsening glycemic control, microvascular complications such as retinopathy and peripheral neuropathy, and significant macrovascular morbidity [2-4]. Medical therapy, when applied effectively, can prevent or delay the development of long-term complications, mediated largely through improved control of blood pressure, glycemia, and lipids $[3,5,6]$.

\footnotetext{
*Correspondence: rgrant@partners.org

1 The Diabetes Center, Massachusetts General Hospital, 50 Staniford Street, Boston MA, 02114, USA

Full list of author information is available at the end of the article
}

Several cross-sectional US national surveys have provided important population-level data on diabetes prevalence and metabolic control over time. Data from the National Health and Nutritional Examination Survey (NHANES), for example, have confirmed an increasing diabetes prevalence, increasing obesity among patients with diabetes, and an apparent trend towards improved metabolic control after years of worsening [7-10]. However, interpretation of these trends is confounded by changes in US demographics and changes in screening criteria over the past decade. One study from the National Health Interview Survey, for example, found that despite significant increases in the diabetes population total each year, the mean age for patients with diabetes remained constant over an 8 year period [11].

Longitudinal studies of defined patient populations, conversely, allow the identification of actual trends in metabolic control over time and can identify individual- 
level predictors of worsening or improved glycemic control over time. We used records from a large hospital network to examine trends in both glycemic and lipid control over a 10-year span. We sought to determine whether changes in care practices would outweigh the impact of ten years of disease progression on glycemic and lipid control in a "real-world" cohort of patients with type 2 diabetes.

\section{Methods}

\section{Setting and Patients}

We used an electronic clinical data query to identify all adults with type 2 diabetes (T2D) receiving outpatient care at one of the 12 outpatient practices affiliated with Massachusetts General Hospital (MGH) in 1996. T2D was defined as 2 outpatient encounters or 1 inpatient encounter with T2D billing code (250.xx) during 1996 or an HbA1c > 7\% prior to 1996. To further refine our T2D population, we excluded patients $<35$ years of age and patients with "Type 1" modifiers in their electronic medical record (EMR) problem lists or ICD-9 billing codes.

We grouped the 4944 eligible patients into 3 cohorts: 1 ) the longitudinal cohort (patients who had at least one HbA1c assay performed in both 1996 and 2006); 2) patients who died before 2006, and 3) the lost to follow up cohort (the remaining patients with no 2006 data and no record of death). The study was approved by the Massachusetts General Hospital/Partners Health Care System Institutional Review Board.

\section{Clinical Variables}

We used a combination of billing, laboratory, EMR, and appointment data sources to collect the following demographic and clinical data: age, gender, race, insurance status, primary language, HbA1c results and number of tests, number of outpatient visits, lipid results, and creatinine results. Vital status was determined using both hospital records and Social Security Administration's Death Master File [12].

Because the electronically-available data available for the overall cohort did not include the more detailed clinical information available from physician narrative progress notes in 1996, we also conducted two structured chart review analyses to address two secondary questions: 1) Among patients with very poor glycemic control in 1996 (HbA1c $\geq 11.5 \%, n=152)$, did patients in the longitudinal cohort $(n=69)$ differ significantly from patients who were lost to follow-up $(n=83)$ in terms of medical and psychiatric comorbidity, substance abuse, or primary language spoken? And, 2) Among patients with moderately poor glycemic control in 1996, were patients who improved over the 10 -year period more likely to receive insulin than patients who worsened? To address this second question, we conducted a 1:1 case-control analysis among the longitudinal cohort of patients with $\mathrm{HbA1c}$ levels between 8.0 and $9.0 \%$ in $1996(\mathrm{n}=394)$ by randomly selecting 50 patients whose HbA1c was at least $1 \%$ lower in 2006 (cases) and 50 patients whose HbA1c was at least 1\% higher in 2006 (controls). These patients were matched by age and gender.

The HbA1c results were obtained using a high-performance liquid chromatography method that has been described previously $[9,13,14]$. This assay serves as one of the primary reference laboratory methods for the National Glycohemoglobin Standardization Program and has inter- and intra-assay coefficients of variation $<2.5 \%$. Long-term drift is prevented by the use of long-term controls. Total plasma cholesterol and plasma triglyceride levels were measured enzymatically [15], high-density lipoprotein fraction was measured after precipitation of low-density, and very low-density lipoproteins were measured using dextran sulfate-magnesium [16]. We estimated LDL cholesterol levels indirectly using the Friedewald formula for patients with plasma triglyceride levels $<400 \mathrm{mg} / \mathrm{dL}$ [17].

\section{Statistical Analysis}

We compared the baseline characteristics of the 3 cohorts using analysis of variance and $\mathrm{t}$-tests for continuous data and chi-square tests for categorical variables. For the longitudinal cohort, we examined individual trends in HbA1c and lipid control between 1996 and 2006. We separately modeled predictors of worsened glycemic control and worsened cholesterol control using multivariate regression modeling after assessing correlations between predictor variables. For the two chart review analyses, we used chi-square tests to compare proportions and report odds ratios. SAS (SAS v 9.1, Cary, North Carolina) was used for all analyses.

\section{Results}

\section{Patient Characteristics}

Of the 4944 eligible patients, 1772 (35.8\%) had HbA1c assays performed at MGH in both 1996 and 2006; 1296 (26.2\%) were lost to follow-up by 2006; and 1876 (37.9\%) died (Table 1). The mean age for all eligible patients in 1996 was 60.0 ( \pm 16.9 ) years, $47.9 \%$ were women, $23.4 \%$ were non-white, and $12.3 \%$ were non-English speaking. The mean HbA1c in 1996 was 8.3\% ( \pm 1.8 ).

The three cohorts varied significantly at baseline by age, sex, race/ethnicity, language, and $\mathrm{HbA1c}$ levels (Table 1). Patients who died in the intervening decade were more than a decade older (mean age $70.6 \pm 10.75$ years) than the other 2 cohorts ( $\mathrm{p}<0.001$ for either comparison). Compared to the longitudinal cohort, the lost to follow-up cohort had a slightly higher mean HbA1c (8.4 \pm $1.9 \%$ vs. $8.2 \pm 1.7 \%, \mathrm{p}=0.002)$ and fewer HbA1c tests $(1.8$ \pm 1.1 vs. $2.1 \pm 1.1, \mathrm{p}<0.001)$ in 1996 . 
Table 1: Patient Characteristics in 1996 by cohort status $(n=4944)$.

\begin{tabular}{|c|c|c|c|c|c|}
\hline & \multirow{2}{*}{$\begin{array}{l}\text { All Patients } \\
(n=4944)\end{array}$} & \multicolumn{4}{|c|}{ Characteristics by cohort } \\
\hline & & $\begin{array}{c}2006 \text { Cohort } \\
(n=1772,35.8 \%)\end{array}$ & $\begin{array}{c}\text { Lost to F/U } \\
(n=129626.2 \%)\end{array}$ & $\begin{array}{c}\text { Died }(n=1876, \\
37.9 \%)\end{array}$ & P-value $^{\dagger}$ \\
\hline Age, years (SD) & $60.0(16.9)$ & $58.8(12.4)$ & $59.7(11.5)$ & $70.6(10.7)$ & $<0.001$ \\
\hline Women & $47.9 \%$ & $49.7 \%$ & $50.2 \%$ & $44.5 \%$ & 0.001 \\
\hline Non-White race/ethnicity & $23.4 \%$ & $21.7 \%$ & $35.7 \%$ & $16.4 \%$ & $<0.001$ \\
\hline Non-English Language & $12.3 \%$ & $10.2 \%$ & $18.3 \%$ & $10.2 \%$ & $<0.001$ \\
\hline Mean HbA1c \% (SD) & $8.3(1.8)$ & $8.2(1.7)$ & $8.4(1.9)$ & $8.3(1.8)$ & 0.008 \\
\hline Mean \# of HbA1c tests (SD) & $2.0(1.1)$ & $2.1(1.1)$ & $1.8(1.1)$ & $1.9(1.1)$ & $<0.001$ \\
\hline Mean \# of encounters (SD) & $13.5(11.8)$ & $12.2(10.7)$ & $11.0(10.4)$ & $16.4(13.9)$ & $<0.001$ \\
\hline \multicolumn{6}{|c|}{$\begin{array}{l}\text { "2006 cohort" = patients with HbA1c results in both } 1996 \text { and } 2006 \text {; "Lost to F/U" = patients who were lost t } \\
=\text { patients who died prior to } 2006 ; \text { SD }=\text { Standard Deviation } \\
\text { † P-values compare } 2006 \text { cohort }(n=1772) \text { to lost-to-follow up cohort }(n=1549) \text { to Died cohort }(n=1902) \\
\text { Mean HbA1c and number of tests and outpatient encounters were those during the calendar year } 1996 .\end{array}$} \\
\hline
\end{tabular}

\section{Glycemic Control in the Longitudinal Cohort}

The mean HbA1c decreased by $0.4 \pm 1.8 \%$ (from $8.2 \% \pm$ $1.7 \%$ to $7.8 \% \pm 1.4 \%$ between 1996 and 2006) among patients in the longitudinal cohort with the majority (58.6\%) having improved their glycemic control over this 10 -year period. The group with the poorest glycemic control in 1996 experienced the biggest decreases in their HbA1c over the 10 years, while the patients with relatively good glycemic control in 1996 (HbA1c $<=8 \%$ ) tended to have increased HbA1c by 2006 (Figure 1). The proportion of patients with $\mathrm{HbA} 1 \mathrm{c}<7.0 \%$ increased from $23.4 \%$ to $26.9 \%$ over the ten-year $\operatorname{span}(\mathrm{p}=0.02)$.

Using logistic regression, we found that higher baseline age (OR 1.41 per decade, 95\% CI: 1.3-1.6, $\mathrm{p}<0.001$ ), higher baseline HbA1c (OR 2.9 per 1\% higher HbA1c, 2.6 - 3.2, p < 0.001), and speaking English (OR 2.1, 1.4-3.1, p < 0.001 ) each independently predicted HbA1c improvement over the next 10 years (Table 2), while race, gender, creatinine and lipid levels, testing and visit frequency did not. A linear regression model with just these three cova-

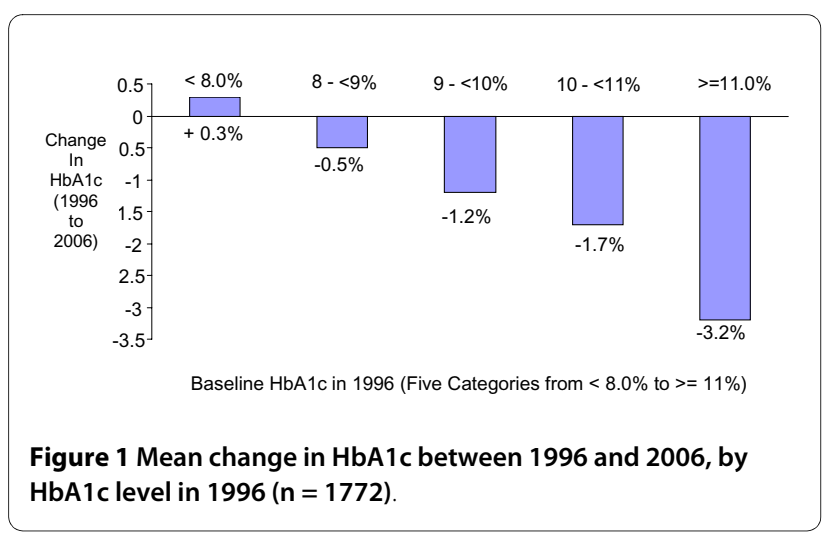

riates explained $51 \%$ of the variability in $\mathrm{HbA} 1 \mathrm{c}$ change over time (adjusted R-squared 0.51).

Figure 2 presents the mean HbA1c per year from 1996 to 2005 for the longitudinal group. This figure also shows mean annual HbA1c levels for the lost to follow-up cohort for as many years as available for each patient. Although baseline HbA1c remained slightly higher from baseline onward in this second group, the improvement trend follows a similar pattern as the longitudinal cohort.

\section{Dyslipidemia in the Longitudinal Cohort}

Total cholesterol results in both 1996 and 2006 were available for 1131 patients $(63.8 \%)$ in the longitudinal cohort. In this subset of patients, $89.1 \%$ of patients had a decrease in total cholesterol and the mean total cholesterol decreased by an average of $49.3( \pm 46.5) \mathrm{mg} / \mathrm{dL}$ between 1996 and 2006. The prevalence of LDL testing increased from $61.5 \%$ in 1996 to $84.1 \%$ in 2006. Of the 945 patients with LDL measurements in both periods, mean levels declined $54.0( \pm 37.7) \mathrm{mg} / \mathrm{dL}$, while HDL levels among patients measured in both periods $(\mathrm{n}=1131)$ increased by $11.6( \pm 11.4) \mathrm{mg} / \mathrm{dL}$. Independently significant baseline predictors of total cholesterol improvement over 10 years included: Older age (OR 1.6 per decade, 95\%CI: 1.3-1.92, $\mathrm{p}<0.001$ ), male gender (OR 2.4 1.6 - 3.6, $\mathrm{p}<0.001$ ), and baseline cholesterol level (OR 1.04 per $\mathrm{mg} / \mathrm{dL}, 1.03-1.04, \mathrm{p}<0.001$ ) (Table 2). In a linear regression model of cholesterol change, these 3 covariates explained $48 \%$ of the model variability (adjusted Rsquared 0.48).

\section{Results of Structured Chart Review Analyses}

Among patients with very poor glycemic control (HbA1c $>11.5 \%)$ at baseline, structured medical chart review revealed that patients lost to follow up $(\mathrm{n}=83)$ were more likely to be drug $(7.2 \%$ vs. $1.5 \%, \mathrm{p}=0.04)$ or alcohol users 
Table 2: Baseline predictors of improved HbA1c or total cholesterol levels from 1996-2006, multivariate logistic regression models.

\begin{tabular}{|c|c|c|c|c|}
\hline & HbA1c $(n=1772)$ & P-Value & $\begin{array}{l}\text { Total Cholesterol } \\
(n=1172)\end{array}$ & P-Value \\
\hline Age (decade) & $1.41(1.3-1.6)$ & $<0.001$ & $1.6(1.3-1.9)$ & $<0.001$ \\
\hline Male gender & 1.1 (1.6 to 3.6$)$ & 0.41 & $2.4(1.6-3.6)$ & $<0.001$ \\
\hline English Speaking & $2.1(1.4-3.1)$ & 0.01 & $1.0(0.5$ to 2.2$)$ & 0.95 \\
\hline 1996 level & $2.9(2.6$ - 3.2) (for HbA1c) & $<0.001$ & $1.04(1.03-1.04)$ (for total cholesterol) & $<0.001$ \\
\hline
\end{tabular}

(12.1\% vs. $2.9 \%, \mathrm{p}=0.02)$, have a language barrier $(22.9 \%$ vs. $8.7 \%, \mathrm{p}=0.05)$, and have no insurance or Medicaid $(40.3 \%$ vs. $26.2 \%, \mathrm{p}=0.08)$ compared to patients who remained in care through to $2006(n=69)$. There were no significant differences in BMI, location of diabetes care, or prevalence of prescribed glycemic, blood pressure, lipid, or psychiatric medications between these case and control patients (data not shown).

In the nested case-control study of patients in the longitudinal cohort with moderately poor control at baseline (HbA1c 8.0-9.0\%) matched by age and gender, we found that patients whose HbA1c significantly improved (cases) were less likely to take insulin in 1996 (40.0\% vs. 66.0\%, p $=0.045)$, have private insurance or Medicare in 1996
(93.0\% vs. $76.6 \%, \mathrm{p}=0.03)$, or attend a diabetes specialty clinic $(26.5 \%$ vs. $46.0 \%, p=0.04$ in $1996 ; 34.0 \%$ vs. $52.0 \%$, $\mathrm{p}=0.06$ in 2006) than those whose HbA1c significantly worsened (controls). The mean weight loss (2.4 lbs vs. 5.3 lbs, $\mathrm{p}=0.6)$ and mean increase in number of prescribed medications ( 4.2 vs. $4.3, \mathrm{p}=0.8$ ) were not significantly different between cases and controls.

\section{Discussion}

The results of this study demonstrate that among patients receiving clinical care in the same setting, glycemic control improved significantly between 1996 and 2006 despite the aging of the cohort and the 10-year increase in duration of their diabetes. Improvement in lipid con-

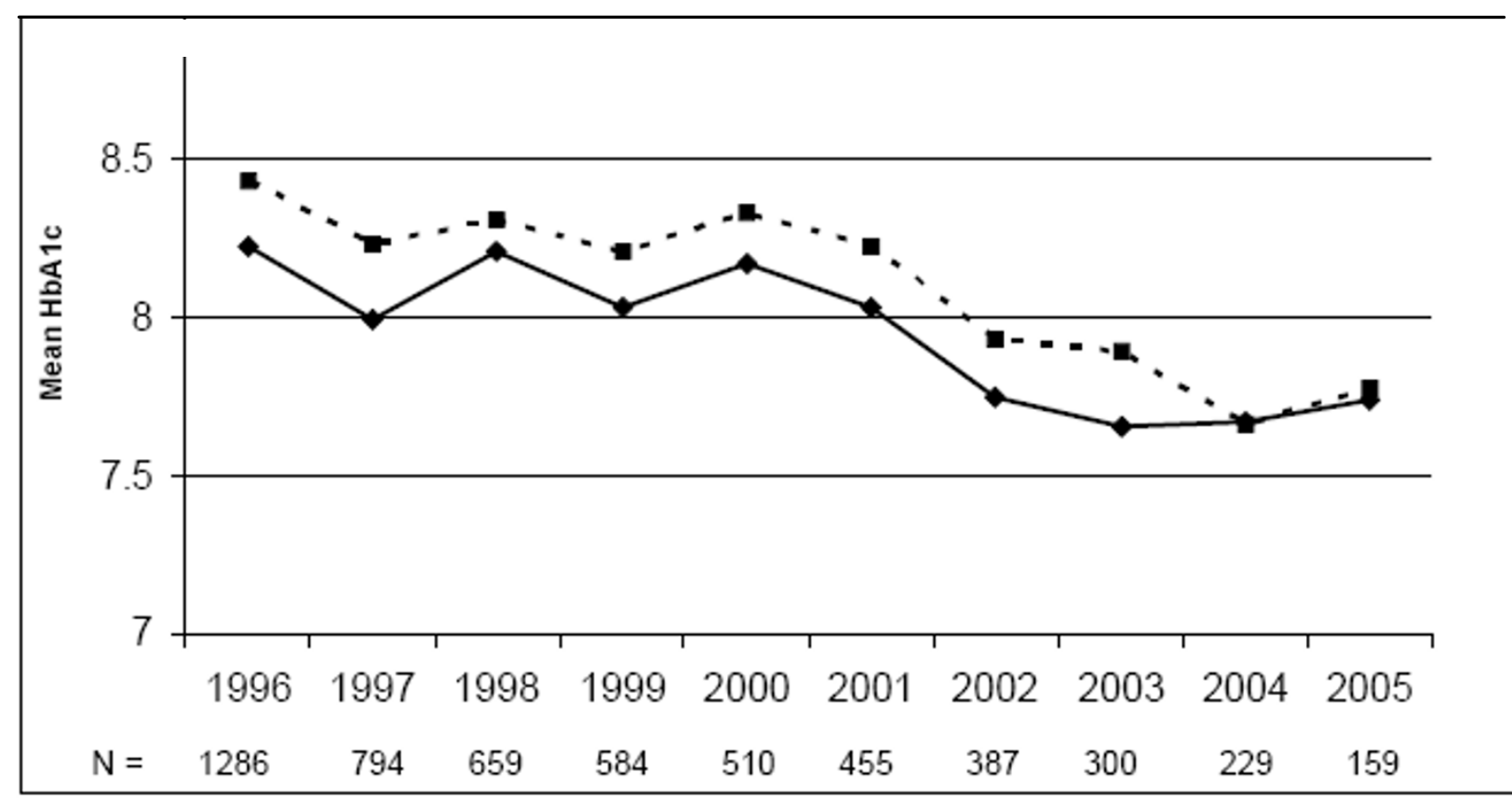

Figure 2 Annual mean HbA1c from 1996-2005, comparing longitudinal (solid line, $n=1772$ in all years) vs. lost to follow-up (dashed line, number if remaining patients for each year provided below graph). 
trol was even more pronounced, likely a consequence of the greater efficacy and ease of pharmacologic treatment for dyslipidemia relative to hyperglycemia. These results support the conclusion that national trends towards improved diabetes-related risk factor control are not solely the result of "down-staging" due to greater screening and earlier diabetes diagnosis, but reflect significantly more effective disease control for individuals living with diabetes.

Three patient factors (older age, higher HbA1c, and speaking English) were strongly predictive of improved HbA1c over time, explaining roughly half the variability in our patient population. The fact that older patients had the most improvement suggests that the later onset phenotype of type 2 diabetes may be more responsive to therapy. These results complement a recent study that found among T2DM patients with Hba1c $<7.0 \%$, older patients were less likely to have worsening glycemic control over the next year than younger patients [18]. Taken together, these results suggest that more aggressive treatment approaches may be needed for younger patients to achieve similar success. It is perhaps not surprising that patients with the highest HbA1c improved the most, since they had the greatest margin for change. These results support therapeutic optimism rather than resignation regarding patients with the poorest control. Finally, the barrier of not speaking English highlights the critical role of effective communication in achieving goals of care over time. It is also possible that speaking English is a proxy for educational level, another factor that may play a role in achieving diabetes control.

A significant proportion of our baseline cohort (26\%) was lost to follow-up. In contrast to the loss to follow-up associated with clinical trials, this loss is reflective of the real world setting in which many patients in our increasingly mobile society (particularly younger and poorer patients) do not remain in the same care setting over time. The demographic differences at baseline underscore the fact that these patients were not lost at random. In our structured chart review, we found that patients with poor control who were subsequently lost to followup were less likely to have private insurance and more likely to have drug or alcohol problems than similarly poorly controlled patients who remained in care. These results suggest that $0.4 \%$ 10-year HbA1c improvement may be an over-estimate, at least among patients with poor baseline control. However, we did see (in Figure 2) similar annual trends of HbA1c improvement among patients ultimately lost to follow-up for the duration of their care in the system after 1996.

The fate of our 1996 cohort also underscores the lethality of diabetes, with over one-third of our patients dying before 2006. Patients who died were demographically dis- tinct from our follow-up cohorts, with significantly older mean age. For the purposes of our analysis, we did not focus further on this group of patients, since our primary goal was to track HbA1c changes over ten-years among patients with a greater than 10-year life expectancy (i.e. the population of patients most likely to benefit from tight glycemic control). Taking the lost to follow-up and mortality cohorts together, nearly two-thirds of patients present in 1996 were not in our system in 2006. Thus, the improvement in $\mathrm{HbA1c}$ control we found over the decade of study may not be generalizable to the overall U.S. population.

The results of this study support the findings in adult participants from the National Health and Nutrition Examination Survey (NHANES). The earlier of the two analyses found that glycemic control rates $(\mathrm{HbA} 1 \mathrm{c}<7 \%)$ declined from $44.5 \%$ in NHANES III (1988-1994) to $35.8 \%$ in NHANES 1999-2000 [8] despite improvements in treatment and understanding of the disease during this period. These initial findings, however, were reversed in the later study, however, suggesting that a significant improvement in glycemic control occurred between 1999 and 2004 [7]. Our results also reveal a similar magnitude of 10-year decline in HbA1c compared to a smaller study conducted in our system between 1985 and 1993 in which HbA1c's in 137 patients with T2DM declined from $8.8 \%$ to $8.4 \%(\mathrm{P}=0.09)$ [9]. Overall, these results demonstrate that at the individual (rather than population) level, patients with T2D have had an incremental decline in $\mathrm{HbA1c}$ despite increasing diabetes duration over the past two decades.

Our results must be interpreted in the context of the study design. Because we included all eligible patients in 1996 (rather than selecting patients based on specific HbA1c levels), our results are not biased by "regression to the mean" seen in other studies that pre-select patients at one of the extremes of the outcome distribution. However, clinical data for our overall patient cohort were limited to electronic medical record, laboratory, and medical billing claims data. While this approach allowed us to efficiently analyze one of the largest reported 10-year "usual care" clinical cohorts for type 2 diabetes, we could not provide specific information about actual medication prescription patterns. To address the need for more detailed data, we performed two structured chart reviews. The analysis of the patients very poorly controlled at baseline $(\mathrm{HbA} 1 \mathrm{c}>11.5 \%)$ provided insight into the social barriers facing patients with poor control who were lost to follow-up (i.e. alcohol and drug use, inadequate insurance, and language discordance). The comparison of patients who improved vs. those who worsened over the next decade revealed higher use of insulin in 1996 among patients who worsened, suggesting 
that insulin use in that earlier era is a strong marker for greater disease severity; conversely, initiation of insulin after 1996 may have been an important factor among those who improved.

\section{Conclusion}

We found that among patients who remained in our health care system, HbA1c levels declined significantly despite ten years of disease progression and patient aging. Improvements in lipid control were even more pronounced and provide corresponding hope that rates of cardiovascular disease may continue to decline in this high-risk population. Older patients with favorable 10year prognoses were more likely to achieve glycemic improvement than younger patients, suggesting that the current treatment of younger patients requires a greater therapeutic emphasis to achieve comparable long-term glycemic and cholesterol benefits as older patients.

\section{Abbreviations}

(T2D): Type 2 diabetes; (NHANES): National Health and Nutritional Examination Survey; (MGH): Massachusetts General Hospital; (HbA1c): Hemoglobin A1c;

\section{Competing interests}

The authors declare that they have no competing interests.

\section{Authors' contributions}

$K L B, M E L, D M N$, and RWG made substantial contributions to conception and design, KJB, MEL, and GW to analysis and interpretation of data; KJB and RWG drafted the manuscript and MEL, GW, DMN revised it critically for important intellectual content; and all authors have given final approval of the version to be published.

\section{Acknowledgements}

Dr. Grant was supported by NIDDK Career Development Award (K23 DK067452). Dr. Nathan, Karen Blumenthal, Mary Larkin and Gail Winning were supported in part by the Earl Charlton Fund for Innovative Diabetes Research.

\section{Author Details}

'The Diabetes Center, Massachusetts General Hospital, 50 Staniford Street, Boston MA, 02114, USA, 2Division of General Medicine, Massachusetts General Hospital, Fruit Street, Boston MA, 02114, USA and ${ }^{3}$ Harvard Medical School, Shattuck Street, Boston, MA 02115, USA

Received: 1 December 2009 Accepted: 9 June 2010 Published: 9 June 2010

\section{References}

1. Center for Disease Control and Prevention: National Diabetes Fact Sheet. In General information and national estimates on diabetes in the United States, 2005 Atlanta, Georgia: U.S. Department of Health and Human Services, Centers for Disease Control and Prevention; 2005.

2. Diabetes Control and Complications Trial Research Group: The effect of intensive treatment of diabetes on the development and progression of long-term complications in insulin-dependent diabetes mellitus. $\mathrm{N}$ Engl J Med 1993, 329:977-986.

3. UK Prospective Diabetes Study (UKPDS) Group: Intensive blood glucose control with sulphonylureas or insulin compared with conventional treatment and risk of complications in patients with type 2 diabetes (UKPDS 33). Lancet 1998, 352:837-853.

4. Brownlee M, Hirsch IB: Glycemic variability: a hemoglobin $A_{1 C^{-}}$ independent risk factor for diabetic complications. JAMA 2006, 295:1707-1708.

5. Heart Protection Study Collaborative Group: MRC/BHF Heart Protection Study of cholesterol-lowering with simvastatin in 5963 people with diabetes: a randomized placebo-controlled trial. Lancet 2003 361(9374):2005-2016

6. Gaede P, Vedel P, Larsen N, Jensen GV, Parving HH, Pedersen O: Multifactorial intervention and cardiovascular disease in patients with type 2 diabetes. N Engl J Med 2003, 348:1925-1927.

7. Ford ES, Li C, Little RR, Mokhad AH: Trends in A1c concentrations among U.S. adults with diagnosed diabetes from 1999 to 2004. Diabetes Care 2008, 31:102-104

8. Koro CE, Bowlin SJ, Bourgeois N, Fedder DO: Glycemic control from 1988 to 2000 among U.S. adults diagnosed with type 2 diabetes. Diabetes Care 2004, 27:17-20.

9. Nathan DM, McKitrick C, Larkin M, Schaffran R, Singer DE: Glycemic control in diabetes mellitus: have changes in therapy made a difference? Am J Med 1996, 100:157-163.

10. Sperl-Hillen $\mathrm{JM}, \mathrm{O}^{\prime}$ Connor PJ: Factors driving diabetes care improvement in a large medical group: ten years of progress. Am J Manag Care 2005, 11:S177-S185.

11. Grant RW, McCarthy EP, Singer DE, Meigs JB: Frequent outpatient contact and decreasing medication affordability in patients with diabetes from 1997 to 2004. Diabetes Care 2006, 29:1386-1388.

12. Hill ME, Rosenwaike I: The social security administration's death master file: the completeness of death reporting at older ages. Social Security Bulletin 2001/2002 64:45-51.

13. Nathan D: Labile glycosylated hemoglobin contributes to hemoglobin A1c as measured by liquid chromatography or electrophoresis. Clin Chem 1981, 27:1261-1263.

14. Nathan DM, Singer DE, Hurxthal K, Goodson JD: The clinical information value of the glycosylated hemoglobin assay. NEngl J Med 1984, 310:341-346.

15. McNamara J, Schaefer E: Automated enzymatic standardized lipid analyses for plasma and lipid lipoprotein fractions. Clin Chim Acta 1987, $166: 1-8$

16. Warnick G, Benderson J, Albers J: Dextran sulfate-magnesium precipitation procedure for quantitation of high-density lipoprotein cholesterol. Clin Chem 1982, 28:1379-1382.

17. Friedewald W, Levy R, Fredrickson D: Estimation of the concentration of low density lipoprotein cholesterol in plasma, without the use of the preparative ultracentrifuge. Clin Chem 1972, 18:499-502.

18. Pani L, Nathan DM, Grant RW: Clinical predictors of disease progression and medication initiation in untreated patients with type 2 diabetes and A1c < 7\%. Diabetes Care 2008, 31:386-390.

Pre-publication history

The pre-publication history for this paper can be accessed here: http://www.biomedcentral.com/1472-6963/10/158/prepub

doi: $10.1186 / 1472-6963-10-158$

Cite this article as: Blumenthal et al., Changes in glycemic control from 1996 to 2006 among adults with type 2 diabetes: a longitudinal cohort study BMC Health Services Research 2010, 10:158

\section{Submit your next manuscript to BioMed Central} and take full advantage of:

- Convenient online submission

- Thorough peer review

- No space constraints or color figure charges

- Immediate publication on acceptance

- Inclusion in PubMed, CAS, Scopus and Google Scholar

- Research which is freely available for redistribution 